\title{
Dialektisch-behaviorale Therapie der PTBS bei Patientinnen mit schwerer Störung der Emotionsregulation
}

\author{
Regina Steil, Clara Dittmann, Simone Matulis, Meike Müller-Engelmann, \\ Kathlen Priebe
}

Geht eine posttraumatische Belastungsstörung zusätzlich einher mit einer Störung der Emotionsregulation, so können traumfokussierende Interventionen wie kognitive Umstrukturierung oder formale Exposition mit Elementen aus der dialektisch-behavioralen Therapie kombiniert werden.

\section{Einleitung}

Frühe traumatische Erfahrungen wie sexuelle oder physische Gewalt in der Kindheit und Jugend haben häufig schwerwiegende Folgen im Erwachsenenalter. Die Betroffenen entwickeln nicht selten eine schwere und chronische Posttraumatische Belastungsstörung (PTBS). Häufig liegen zusätzliche Symptome vor wie eine Störung der Emotionsregulation, negatives Selbstkonzept, dissoziative Symptome sowie zwischenmenschliche Probleme.

Viele dieser Patientinnen erfüllen auch die Kriterien für eine komorbide Störung, wie z.B. Substanzmissbrauch oder eine Borderline-Persönlichkeitsstörung (BPS).

Patientinnen mit PTBS und komorbider BPS weisen oft eine große Anzahl an Suizidversuchen, hohe Hospitalisierungsraten und geringere Remissionsraten auf [1 -3$]$.

In der Vergangenheit fehlten auf die Bedürfnisse und Besonderheiten dieser Patientengruppe angepasste evidenzbasierte Behandlungsprogramme.

Aus den Ergebnissen großer Metaanalysen kann nicht geschlossen werden, dass PTBS-Standardbehandlungen für diese Patientengruppe sicher und effektiv sind, weil sie in den bislang vorliegenden Psychotherapiestudien unterrepräsentiert ist (vgl. [4]).
Viele ambulante Behandler haben Sorge, dass der Einsatz der - von allen Leitlinien empfohlenen - traumafokussierten Psychotherapieverfahren kurzfristig zu einer Symptomverschlimmerung oder zu suizidalen Krisen bei diesen Patientinnen führen könnte (vgl. [5]). Häufig wird von Behandlern für den Beginn einer traumafokussierten Intervention verlangt, dass die Patientinnen lange Zeit kein suizidales oder selbstverletzendes Verhalten gezeigt haben. Daher werden die Patientinnen leider häufig (auch lange) psychotherapeutisch behandelt, ohne überhaupt jemals gezielt über die traumatischen Erfahrungen zu sprechen [6].

Entwicklung der DBT-PTSD. Eine deutsche Arbeitsgruppe um M. Bohus und R. Steil entwickelte in den letzten 10 Jahren eine spezifische Psychotherapie für die PTBS mit schwerer Störung der Emotionsregulation (wie z.B. und v.a. eine komorbide Borderline-Persönlichkeitsstörung): die Dialektisch-behaviorale Therapie der PTBS (DBT-PTSD) [7, 8]. Diese Behandlung integriert Elemente der Dialektisch-behavioralen Therapie (DBT), klassische kognitiv-behaviorale sowie innovative Interventionen. 


\section{Störungsbild der PTBS}

\section{Definition}

\section{Traumatisierung nach DSM-5}

Die Konfrontation mit Tod oder Lebensbedrohung, ernsthafter Verletzung oder sexueller Gewalt entweder durch direktes Erleben oder Zeugenschaft oder das Erfahren, dass das Ereignis oder die Ereignisse einem Verwandten oder Freund passierten oder die wiederholte Konfrontation mit aversiven Details des Ereignisses oder der Ereignisse.

Für das Stellen der Diagnose der PTBS ist das Erleben eines traumatischen Ereignisses eine Voraussetzung.

Kernsymptome. Die PTBS ist gekennzeichnet durch

- Symptome des Wiedererlebens der belastenden Erinnerungen im Wachen und Schlafen (Intrusionen),

- dysfunktionale Gedanken und Überzeugungen zum Trauma und seinen Folgen,

- emotionale Taubheit,

- Hypervigilanz,

- Übererregung und

- Vermeidungsverhalten.

Die Hauptsymptome der PTBS sind Intrusionen, Vermeidungsverhalten, traumaspezifische dysfunktionale Kognitionen und Übererregung.

PTBS im DSM-5. Eine Vielzahl an Stimuli kann das traumaassoziierte Netzwerk aktivieren und damit Gefühle, Körperempfindungen und Gedanken auslösen, die während der Traumatisierung eine Rolle gespielt haben. Die Patientinnen versuchen, diese Stimuli vermehrt zu vermeiden und entwickeln Strategien wie Grübeln, selbstverletzendes Verhalten oder Substanzkonsum, um die belastenden Gefühle zu beenden.

Im 2013 erschienenen Diagnostic and Statistical Manual of Mental Disorders 5 (DSM-5) wird die PTBS erstmals nicht mehr unter dem Kapitel der Angststörungen aufgeführt, sondern zusammen mit der akuten Belastungsstörung und den Anpassungsstörungen im Kapitel „Trauma- and Stressor-Related Disorders“ [9].

Neu ist auch ein stärkerer Fokus auf negative Veränderungen in Kognitionen und Stimmung im Zusammenhang mit dem traumatischen Ereignis wie anhaltende und übertriebene negative Überzeugungen oder Erwartungen in Bezug auf sich selbst, andere oder die Welt (z. B. „Ich bin schlecht“, „Man kann niemandem vertrauen“, „Die Welt ist total gefährlich“, „Mein ganzes Nervensystem ist dauerhaft ruiniert“) (s. Tab.1).

Im DSM-5 wird erstmals zusätzlich ein Kriterium zu negativen Veränderungen in Kognitionen und Stimmung angegeben.

Komplexe Traumatisierung. Nach multipler Traumatisierung in Kindheit und Jugend entwickeln Patientinnen häufig zusätzlich zu der PTBS-Kernsymptomatik Störungen in den Bereichen:

- Emotionsregulation

- interpersonelle Fertigkeiten

- Aufmerksamkeit und Bewusstsein

- Glaubenssystem

- somatische Symptome [10].

Diese Patientinnen haben aufgrund von frühen negativen Erfahrungen häufig keine ausreichenden Fertigkeiten, mit ihren starken Emotionen umzugehen, und nutzen dysfunktionale, langfristig schädliche Bewältigungsstrategien zur Emotionsvermeidung wie Selbstverletzung, Drogen- oder Alkoholmissbrauch oder Hochrisikoverhalten.

Mit der International Classification of Diseases 11 (ICD-11) wird voraussichtlich ein neues Störungsbild der komplexen PTBS eingeführt werden, welches im Gesundheitswesen die besondere Schwere und über die PTBS hinausgehende Symptomatik diagnostizierbar macht (vgl. [11]).

\section{Bisherige Therapieansätze und deren Wirksamkeit}

\section{Metaanalysen}

In Metaanalysen zu Psychotherapiestudien der PTBS konnten für traumafokussierende kognitiv-behaviorale Behandlungsverfahren und Eye Movement Desensitization and Reprocessing (EMDR) große Effekte auf die posttraumatische Symptomatik ermittelt werden [12 14]. Inwieweit diese Ergebnisse auf die Behandlung der PTBS mit schwerer Störung der Emotionsregulation übertragen werden können, ist jedoch unklar. Denn in vielen Studien wurden Patientinnen mit Symptomen wie z.B. Suizidalität oder selbstverletzendes Verhalten ausgeschlossen. Beispielsweise wurden in der Metaanalyse von Bradley et al. zu 26 Behandlungsstudien Patientinnen mit Suizidrisiko in $46 \%$ der Studien aus- 


\section{Tabelle 1}

DSM-5-Kriterien der Posttraumatischen Belastungsstörung (309.81) [9].

\begin{tabular}{|c|c|}
\hline A & $\begin{array}{l}\text { Konfrontation mit Tod oder Lebensbedrohung, ernsthafter Verletzung oder sexueller Gewalt auf eine oder mehrere } \\
\text { der folgenden Arten: } \\
\text { - direktes Erleben } \\
\text { - persönliches Miterleben, wie das Ereignis einer anderen Person passiert } \\
\text { - Erfahren, dass das Ereignis einem Verwandten oder einem Freund passierte } \\
\text { - wiederholte Konfrontation mit aversiven Details des Ereignisses }\end{array}$ \\
\hline B & $\begin{array}{l}\text { Intrusive Symptome nach dem Ereignis (mind. 1) } \\
\text { - Erinnerungen } \\
\text { - (Alb-)Träume } \\
\text { - psychische Belastung bei Konfrontation mit traumassoziierten Reizen } \\
\text { - physiologische Reaktion bei Konfrontation mit traumaassoziierten Reizen }\end{array}$ \\
\hline C & $\begin{array}{l}\text { Anhaltende Vermeidung von traumassoziierten Reizen (mind. 1) } \\
\text { - Vermeidung von Erinnerungen, Gedanken oder Gefühlen } \\
\text { - Vermeidung von äußeren Hinweisreizen (Orte, Menschen ...) }\end{array}$ \\
\hline D & $\begin{array}{l}\text { Negative Veränderungen in Kognitionen und Stimmung im Zusammenhang mit dem Ereignis (mind. 2) } \\
\text { - Unfähigkeit Traumaspekte zu erinnern } \\
\text { - anhaltende und übertriebene, negative Grundüberzeugungen; verzerrte Schuldzuweisungen } \\
\text { - negativer Gefühlszustand (z. B. Angst, Scham, Schuld) } \\
\text { - Interessenverlust und Reduktion von Aktivitäten } \\
\text { - Gefühl der Distanziertheit und Entfremdung von anderen } \\
\text { - Unfähigkeit, positive Gefühle zu empfinden }\end{array}$ \\
\hline E & $\begin{array}{l}\text { Veränderung hinsichtlich Arousal und Reagibiliät im Zusammenhang mit dem Ereignis (mind. 2) } \\
\text { - Reizbarkeit/Wutausbrüche } \\
\text { - rücksichtsloses, selbstdestruktives Verhalten } \\
\text { - Hypervigilanz } \\
\text { - übertriebene Schreckreaktion } \\
\text { - Konzentrationsstörungen } \\
\text { - Schlafstörungen }\end{array}$ \\
\hline $\mathbf{F}$ & Das Störungsbild dauert länger als einen Monat. \\
\hline G & Das Störungsbild verursacht klinisch bedeutsames Leiden und Beeinträchtigungen in wichtigen Bereichen. \\
\hline & nz oder eine medizinische Ursache. \\
\hline
\end{tabular}

geschlossen, solche mit Abhängigkeitserkrankungen und mit schwerer Komorbidität jeweils in 62\% der Studien [12]. Es ist wahrscheinlich, dass diese Ausschlusskriterien häufiger auf Patientinnen mit PTBS mit schwerer Störung der Emotionsregulation zutreffen.

In den wenigen vorliegenden Studien, die den Einfluss einer komorbiden BPS auf das Behandlungsergebnis einer PTBS untersucht haben, findet sich zwar eine vergleichbare Reduktion der posttraumatischen Symptome nach traumafokussierender kognitiver Verhaltenstherapie bei Patientinnen ohne BPS im Vergleich zu denen mit BPS [15-17]; es gilt jedoch zu berücksichtigen, dass Patientinnen mit gegenwärtigem selbstverletzendem Verhalten ausgeschlossen wurden.
Zudem wiesen die Patientinnen mit BPS ein geringeres Endfunktionsniveau auf. In einer randomisierten kontrollierten Studie zur Behandlung der PTBS nach Kindesmissbrauch, welche spezifische Informationen zur Wirksamkeit bei Patientinnen mit einer BPS beinhaltet, zeigte sich, dass alle Patientinnen mit einer komorbiden BPS eine primär expositionsbasierte Therapie abbrachen [18].

Die Wirksamkeit bislang etablierter Verfahren bei PTBS mit schwerer Störung der Emotionsregulation ist unklar. 


\section{Berücksichtigung in nationalen und inter- nationalen Leitlinien}

Die Unsicherheit zur Behandlung der PTBS mit komorbiden Störungen wird auch in Behandlungsleitlinien der PTBS deutlich.

ISTSS-Leitlinien. In den Leitlinien der International Society for Traumatic Stress Studies (ISTSS) wird generell eine traumafokussierende kognitiv-behaviorale Behandlung empfohlen; es wird jedoch angeführt, dass für Patientinnen mit komorbiden dissoziativen Störungen und Persönlichkeitsstörungen bislang nur wenige Daten vorliegen [19].

APA-Leitlinien. Die Leitlinien der American Psychiatric Association (APA) betonen, dass Suizidalität und selbstverletzendes Verhalten bei der Behandlungsplanung zu berücksichtigen sind und bei komorbiden Störungen eine längere Sitzungszahl notwendig sein kann $[20,21]$.

NICE-Leitlinien. Die britischen NICE-Leitlinien führen an, dass Abhängigkeitserkrankungen und Suizidalität vor der PTBS-Behandlung fokussiert werden sollten [22]. Zudem wird für Patientinnen mit multiplen Traumatisierungen und schweren komorbiden Störungen eine größere Sitzungszahl eingeräumt.

Australische Leitlinien. In den australischen Leitlinien wird angeführt, dass bei komplexen Störungsformen häufig zusätzliche Sitzungen, ein Training der Emotionsregulation sowie ein graduiertes Vorgehen in der Exposition notwendig sind [23].

AWMF-Leitlinien. In der deutschsprachigen Leitlinie der Arbeitsgemeinschaft der Wissenschaftlichen Medizinischen Fachgesellschaften (AWMF) wird die traumafokussierende Therapie generell auch bei komplexer PTBS, also bei Patientinnen mit schwerer Störung der Emotionsregulation, als Methode der Wahl aufgeführt [24]. Dabei sollten Probleme der Affektregulation in der Behandlungsplanung initial berücksichtigt werden.

Die in vielen der Leitlinien erwähnte Schwierigkeit der Generalisierung der Ergebnisse auf Patientinnen mit komplexer PTBS hat die ISTSS veranlasst, eine Expertenbefragung zur komplexen PTBS durchzuführen [25]. Als typischstes Merkmal einer komplexen PTBS wurde die Emotionsregulationsstörung herausgestellt.

Als effektivste Interventionen wurden ein Training der Emotionsregulation und das Berichten der traumatischen Erinnerung eingeschätzt.
Studien. Die Arbeitsgruppe um Cloitre hat eine 2-phasige Behandlung - bestehend aus 8 Sitzungen Emotionsregulationstraining und nachfolgenden 8 Sitzungen Exposition - in 2 randomisiert kontrollierten Studien untersucht [26, 27]. Die Wirksamkeit für Patientinnen mit komorbider BPS ist jedoch bislang unklar. In der ersten Studie wurde diese Patientengruppe ausgeschlossen. In der 2010 publizierten Studie wird keine Subgruppenanalyse zur Wirksamkeit bei Patientinnen mit einer komorbiden BPS (24\% der Stichprobe) berichtet.

Als das empirisch am besten gesicherte Konzept zur Behandlung der BPS gilt derzeit die DBT [28]. Der Großteil der Publikationen bezieht sich jedoch auf die Behandlung in Phase I, in der auf die Verbesserung der Emotionsregulation fokussiert wird und v.a. eine Reduktion von suizidalem und selbstschädigendem Verhalten sowie der Hospitalisierung angestrebt wird (Standard-DBT). Die wenigen vorhandenen Daten sprechen dafür, dass die Elemente dieser Phase bei der Mehrheit der BPS-Patientinnen mit komorbider PTBS nicht ausreichen. So berichten Harned und Linehan nach einjähriger ambulanter Standard-DBT eine Remissionsrate der PTBS von nur 13\% [29]. Obwohl im ursprünglichen DBT-Manual die Reduktion posttraumatischer Symptome als wesentliches Ziel der Behandlungsphase II benannt wurde, wurde im Manual das genaue Vorgehen nicht spezifiziert [30].

In den letzten Jahren wurde die Behandlung in Phase II von 2 Arbeitsgruppen entwickelt und erforscht. So liegen nun 2 Behandlungsprogramme vor, für die es erste Wirksamkeitsnachweise gibt:

- Dialektisch-behaviorale Therapie der Posttraumatischen Belastungsstörung (DBT-PTSD)

- Dialektisch-behaviorale Therapie plus Prolongierte Exposition (DBT+PE)

In der DBT+PE erhalten Patientinnen während einer einjährigen Standard-DBT zusätzlich traumafokussierende Expositionssitzungen, sobald sie für mindestens 2 Monate selbstschädigendes und therapiestörendes Verhalten eingestellt haben. In einer amerikanischen Prä-Post-Studie an 13 Patientinnen fand sich eine deutliche Verbesserung der posttraumatischen Symptomatik mit einer Effektstärke von 1,4 [31]. In einer kürzlich publizierten randomisiert kontrollierten Studie, in der DBT+ PE mit Standard-DBT verglichen wurde, fand sich bei beiden eine deutliche Verbesserung der posttraumatischen Symptomatik (DBT + PE g = 1,8; DBT $g=1,3$ ) [32]. Von insgesamt 17 Patientinnen in der DBT + PE-Bedingung begannen jedoch insgesamt nur 
8 Patientinnen - weniger als die Hälfte - überhaupt die traumaspezifische Behandlung.

\section{Grundlagen und Beschreibung der DBT-PTSD}

\section{Anwendungsbereich und Voraussetzungen}

Die DBT-PTSD wurde zunächst für das stationäre Setting entwickelt und hinsichtlich ihrer Wirksamkeit überprüft. Sie erwies sich jedoch auch bei der Übertragung in den ambulanten Bereich als vielversprechend. Im stationären Setting sind 12 Wochen mit 24 Einzelsitzungen angemessen, im ambulanten Rahmen werden ca. 45 Behandlungssitzungen verteilt auf mindestens ein Jahr empfohlen.

Voraussetzung für die stationäre und ambulante Behandlung ist, dass die Patientinnen keine psychotische, bipolare oder demenzielle Erkrankung haben. Zudem darf keine aktuelle Substanzabhängigkeit vorliegen und der Body-Mass-Index sollte über 16 liegen, sonst wäre eine Behandlung in einem anderen Setting bzw. mit einem anderen Schwerpunkt indiziert. Eventuell können auch schwere körperliche (z.B. kardiovaskuläre) Erkrankungen einen Ausschlussgrund darstellen dies muss im Einzelfall in Abstimmung mit Körpermedizinern geprüft werden. Des Weiteren sollte in den letzten 2 Monaten vor Aufnahme der Behandlung kein Suizidversuch, keine lebensbedrohliche Selbstverletzung und kein lebensgefährliches Hochrisikoverhalten stattgefunden haben.

\section{Voraussetzungen für DBT-PTSD}

In den letzten 2 Monaten kein Suizidversuch, keine lebensbedrohliche Selbstverletzung und kein lebensgefährliches Hochrisikoverhalten

\section{Kontraindikation}

- psychotische, biploare oder demenzielle Erkrankung

- Alkohol- und Substanzabhängigkeit

- Body-Mass-Index $<16$

\section{Behandlungshierarchie}

Der Behandlung der DBT-PTSD liegt eine für DBTInterventionen typische dynamische Behandlungshierarchie zugrunde (vgl. Tab.2). Dabei folgt die Behandlung einem klaren Algorithmus, der dem Therapeuten

\section{Tabelle 2}

Dynamische Behandlungshierarchie der DBT-PTSD.

\begin{tabular}{|c|c|c|}
\hline Hierarchiestufen & Behandlungsfokus & Beispiel \\
\hline \multicolumn{3}{|l|}{ Standard-DBT } \\
\hline Stufe 1 & $\begin{array}{l}\text { lebensbedrohliche } \\
\text { Verhaltensweisen }\end{array}$ & Suizidversuche \\
\hline Stufe 2 & $\begin{array}{l}\text { therapiestörende } \\
\text { Verhaltensweisen }\end{array}$ & $\begin{array}{l}\text { Termine nicht wahrnehmen, } \\
\text { nicht antworten }\end{array}$ \\
\hline Stufe 3 & $\begin{array}{l}\text { krisengenerierende } \\
\text { Verhaltensweisen }\end{array}$ & Obdachlosigkeit \\
\hline \multicolumn{3}{|l|}{ DBT-PTSD } \\
\hline Stufe 1 & $\begin{array}{l}\text { Therapiefortschritt } \\
\text { behinderndes Verhal- } \\
\text { ten }\end{array}$ & $\begin{array}{l}\text { dissoziative Symptomatik, } \\
\text { schwere Essstörung }\end{array}$ \\
\hline Stufe 2 & $\begin{array}{l}\text { schwere psychosoziale } \\
\text { Probleme }\end{array}$ & $\begin{array}{l}\text { fatale Partnerschaft, massive } \\
\text { Geldsorgen }\end{array}$ \\
\hline Stufe 3 & $\begin{array}{l}\text { PTBS aufrechterhalten- } \\
\text { des Verhalten }\end{array}$ & $\begin{array}{l}\text { dysfunktionale Escape- und Mei- } \\
\text { dungsstrategien z. B. Vermeidung } \\
\text { von Triggern, ungünstige trauma- } \\
\text { bezogene Kognitionen }\end{array}$ \\
\hline Stufe 4 & $\begin{array}{l}\text { Lebensqualität ein- } \\
\text { schränkendes Verhal- } \\
\text { ten }\end{array}$ & $\begin{array}{l}\text { Ablehnung des eigenen Körpers } \\
\text { sowie der eigenen Sexualität, } \\
\text { gestörte soziale Beziehungen }\end{array}$ \\
\hline
\end{tabular}

bzw. dem behandelnden Team hilft zu entscheiden, in welcher Reihenfolge problematische Verhaltensweisen oder Störungsbereiche fokussiert und behandelt werden sollen.

\begin{abstract}
Abbau von lebensbedrohlichem oder therapiegefährdendem Problemverhalten. Im Fokus stehen zunächst lebensbedrohliche Verhaltensweisen wie Suizidversuche, lebensgefährliche Selbstverletzungen und schweres Hochrisikoverhalten. Anschließend wird daran gearbeitet, Verhaltensweisen zu reduzieren, welche die Therapie gefährden. Hierzu gehören häufiges Zuspätkommen, Nichtwahrnehmen von vereinbarten Terminen und eine fehlende Bereitschaft, sich auf die Behandlung einzulassen. Vor den traumafokussierenden Interventionen sollten zudem krisengenerierende Verhaltensweisen wie drohende Obdachlosigkeit, finanzielles Chaos oder schwere Aggressivität reduziert werden.
\end{abstract}

Bevor mit der formalen Expositionsphase begonnen werden kann, sieht die DBT-PTSD eine Reduktion von 
Verhaltensweisen vor, die den Therapiefortschritt und damit die Wirksamkeit der traumafokussierten Interventionen beeinträchtigen. Hierzu gehören dissoziative Symptome und der Konsum von Substanzen, die die Lernfähigkeit beeinträchtigen, wie z.B. Cannabis.

\section{Das Kernelement der DBT-PTSD ist die formale Exposition.}

Expositionstherapie. Die Hierarchisierung der Behandlung hat das Ziel, auch bei schwer kranken Patientinnen möglichst bald mit der formalen Exposition beginnen zu können, die das Kernelement der Behandlung darstellt. Für den Beginn der Exposition ist es nicht ausgeschlossen, dass z. B. leichte Selbstverletzungen weiterhin vorkommen. Wenn jedoch im Verlauf der Behandlung problematische Verhaltensweisen auftreten, die einer höheren Hierarchie-Ebene zuzuordnen sind, rücken diese unmittelbar in den Fokus der therapeutischen Aufmerksamkeit. So müssen etwa schwere Selbstverletzungen, die auftreten, nachdem bereits mit der Exposition begonnen wurde, vorrangig mithilfe von Verhaltensanalysen bearbeitet werden.

\section{Die Behandlungsphasen der DBT-PTSD}

Die DBT-PTSD besteht aus 5 Behandlungsphasen, die nacheinander durchlaufen werden (Tab.3). Einige Module davon sollten bei allen Patientinnen durchgeführt werden, z.B. die Vermittlung von Skills zum Umgang mit Belastung und die Durchführung der Exposition, während auf andere in Abhängigkeit von den Bedürfnissen der Patientin zurückgegriffen werden kann, z.B. auf die Bearbeitung von Wut und Albtraumbehandlung.

\section{Tipp für die Praxis}

Während der gesamten Behandlung bleibt die oben beschriebene Behandlungshierarchie bestehen, sodass lebensgefährliches, krisengenerierendes und therapiestörendes Verhalten zu jedem Zeitpunkt vorrangig behandelt werden können.

\section{Die Commitmentphase}

In der Commitmentphase findet zunächst eine ausführliche Anamnese statt, in der die aktuelle Lebenssituation, der biografische Hintergrund, Vorbehand-

\section{Tabelle 3}

Die 5 Behandlungsphasen der DBT-PTSD.

\begin{tabular}{|c|c|}
\hline $\begin{array}{l}\text { Behandlungs- } \\
\text { phasen }\end{array}$ & Module \\
\hline $\begin{array}{l}\text { Phase } 1 \\
\text { Commitment }\end{array}$ & $\begin{array}{l}\text { - Anamnese und Psychopathologie } \\
\text { - Exploration von derzeitigem krisen- } \\
\text { generierendem Verhalten } \\
\text { - Therapieaufklärung, Zielklärung } \\
\text { und Therapievertrag }\end{array}$ \\
\hline $\begin{array}{l}\text { Phase } 2 \\
\text { Planung und } \\
\text { Motivation }\end{array}$ & $\begin{array}{l}\text { - PTSD-Störungsmodell } \\
\text { - Befürchtungen } \\
\text { - VA zu Problemverhalten } \\
\text { - Entscheidung für neuen Weg und } \\
\text { Therapieziele }\end{array}$ \\
\hline $\begin{array}{l}\text { Phase } 3 \\
\text { Bearbeitung } \\
\text { von traumabe- } \\
\text { zogenen Esca- } \\
\text { pe-Strategien }\end{array}$ & $\begin{array}{l}\text { - Belastungstoleranz und Umgang mit } \\
\text { Dissoziation } \\
\text { - Gefühlsregulation } \\
\text { - individuelle Bearbeitung von Schuld, } \\
\text { Scham und Wut } \\
\text { - Vorbereitung zur Durchführung der } \\
\text { Exposition }\end{array}$ \\
\hline $\begin{array}{l}\text { Phase } 4 \\
\text { Exposition: } \\
\text { Reduktion des } \\
\text { Wiedererlebens } \\
\text { traumabezoge- } \\
\text { ner Gefühle }\end{array}$ & $\begin{array}{l}\text { - Diskriminationstraining } \\
\text { - Skills-assistierte Exposition } \\
\text { - Albtraumbehandlung } \\
\text { - radikales Annehmen der Vergangen- } \\
\text { heit }\end{array}$ \\
\hline $\begin{array}{l}\text { Phase } 5 \\
\text { Entfaltung des } \\
\text { Lebens }\end{array}$ & $\begin{array}{l}\text { - Selbstwert und Selbstkonzepte } \\
\text { - Reviktimisierung } \\
\text { - Körper, Sexualität und Partnerschaft } \\
\text { - soziales Netzwerk und berufliche } \\
\text { Tätigkeit } \\
\text { - Rückfallprophylaxe und Abschied }\end{array}$ \\
\hline
\end{tabular}

lungen und die bestehenden Symptome erfasst werden. Darüber hinaus exploriert der Therapeut selbstschädigende Verhaltensweisen, Suizidversuche und frühere Therapieabbrüche genau und leitet Verhaltensanalysen hierzu an, in denen die Patientin die prädisponierenden Bedingungen wie auch die nachfolgenden Konsequenzen von schädlichem Verhalten genau analysiert. Zur Erfassung relevanter belastender wie auch positiver biografischer Ereignisse wird eine individuelle Lebenslinie erstellt.

In dieser Phase werden außerdem erste Ziele für die Behandlung definiert und die Patientinnen erhalten eine Einführung in das Skills-Training, bei der sie ein computerbasiertes Selbsthilfe-Programm unterstützt [33]. Ein Schwerpunkt wird auch auf Achtsamkeitsübungen gelegt, die den Patientinnen die Fähigkeit 
vermitteln sollen, in der Gegenwart zu bleiben und zu unangenehmen Erinnerungen und Gefühlen inneren Abstand zu gewinnen. Am Ende der Commitmentphase unterschreiben Patientin und Therapeut den behandlungsspezifischen Therapievertrag.

In der Commitmentphase wird eine individuelle Lebenslinie erarbeitet, mit dem Skills-Training begonnen und der Therapievertrag geschlossen.

\section{Die Planungs- und Motivationsphase}

Viele Patientinnen äußern zu Beginn der Behandlung zunächst v.a. den Wunsch, von den bestehenden Symptomen befreit zu werden. Da die DBT-PTSD die Patientin dabei unterstützen will, trotz der traumatischen Erfahrungen ein erfülltes Leben zu leben, werden der alte Weg (Leben mit den Symptomen) und der neue Weg (z.B. Einsatz mit Skills anstatt Nutzen ungünstiger Escape-Strategien) der Patientin so konkret wie möglich besprochen.

Als nächstes wird ein individuelles Störungsmodell der PTBS erarbeitet.

\section{Tipp für die Praxis}

Für die Erarbeitung des individuellen Störungsmodells greift die DBT-PTSD auf das Bild eines Wasserballs zurück, der durch die Vermeidung der Erinnerungen unter Wasser gehalten wird und bei der kleinsten Unaufmerksamkeit mit Wucht in Form von belastenden Erinnerungen nach oben schießt [34].

Im Rahmen der Entwicklung des Störungsmodells werden individuelle Meidungs- und Escape-Strategien der Patientin erarbeitet und die Befürchtungen der Patientin bezüglich der Aufgabe dieser Strategien (z.B. „Ich könnte verrückt werden“ oder „Ich werde nie mehr aufhören zu weinen“) mithilfe kognitiver Methoden relativiert.

In der Planungs- und Motivationsphase erarbeitet das therapeutische Team ein individuelles Störungsmodell und trifft nach der Bearbeitung von Befürchtungen die Entscheidung für die aktive Auseinandersetzung mit den Erinnerungen.

\section{Die Bearbeitung von traumabezogenen Escape-Strategien}

Skills zur Stresstoleranz und gegen Dissoziation. In dieser Phase der Behandlung erlernen die Patientinnen Stresstoleranzskills, die ihnen helfen sollen, Zustände hoher Anspannung und dissoziative Symptome zu reduzieren (vgl. [33]). Zur Reduktion dissoziativer Symptome werden beispielsweise deren kurz- und langfristige Vor- und Nachteile erarbeitet, Dissoziationsprotokolle geführt, Frühwarnzeichen erarbeitet und antidissoziative Skills vermittelt, wie z.B. der Einsatz von Chilischoten. Darüber hinaus wird die emotionale Verwundbarkeit durch ausreichendes Trinken und Schlafen sowie regelmäßige Bewegung reduziert.

Escape-Strategien bearbeiten. Nach der Verringerung von Escape-Strategien auf der Verhaltensebene, z. B. Dissoziation und Missbrauch von Alkohol, werden die individuellen Escape-Strategien auf der emotionalen Ebene (in der Regel Schuld- oder Wutgefühle) fokussiert.

Schuldgefühle werden reduziert durch den Einsatz kognitiver Interventionen, wie z. B. der Frage, warum die Patientin sich damals so und nicht anderes verhalten hat und das Abwägen der Handlungsmöglichkeiten eines Kindes diesen Alters.

Darüber hinaus kann die Funktion der Schuld erarbeitet werden, wobei sich meist zeigt, dass diese dazu dient, die Illusion einer gewissen Kontrolle über die damalige Situation aufrechtzuerhalten, was meist leichter auszuhalten ist, als das eigentlich zur Situation gehörige Gefühl der Hilflosigkeit.

Zur Reduktion von Schuldgefühlen werden verschiedene kognitive Interventionen wie die Advocatus-diaboli-Technik eingesetzt. Dabei nutzt die Therapeutin die Ambivalenz der Patientin bzgl. der Schuldüberzeugung.

\section{Tipp für die Praxis}

Zur Reduktion von Schamgefühlen, wie sie v.a. bei Opfern sexueller Gewalt häufig vorkommen, ist die Veränderung der Körperhaltung hin zu einer der Scham entgegengesetzten Haltung (z. B. aufrichten und Blickkontakt suchen) besonders wirkungsvoll und hilfreich. 


\section{Die Expositionsphase}

Vorbedingungen für den Einsatz formaler Exposition. Die DBT-PTSD definiert bestimmte Bedingungen, die für den Beginn der formalen Exposition erfüllt sein müssen. Hierzu gehört beispielsweise, dass die Patientin in den letzten Wochen keinen Suizidversuch begangen und keine lebensbedrohlichen Selbstverletzungen oder lebensbedrohliches Hochrisikoverhalten gezeigt hat. Darüber hinaus sollte sie Skills selbstständig einsetzen, Dissoziation entgegensteuern und sich rational von Schuld distanzieren können sowie die Entscheidung für die Exposition getroffen haben.

Die DBT-PTSD definiert bestimmte Bedingungen, die erfüllt sein müssen, bevor mit der formalen Exposition begonnen werden kann.

\section{Tipp für die Praxis}

Die Patientin sollte darauf vorbereitet werden, dass die nächsten Wochen mit einer vermehrten Belastung einhergehen können. Aus diesem Grund sollte die Patientin eine Person aus ihrem Umfeld haben, die sie während der Exposition unterstützen kann und die der Therapeut bei Bedarf vor Beginn der Exposition zu einem gemeinsamen Gespräch einlädt.

Durch Kontextlernen und Elaboration des TraumaGedächtnisses kommt es im Verlauf der Exposition zu einer Reduktion der belastenden Gefühle - aus einem unkontrollierten Wiedererleben soll ein kontrollierbares Erinnern werden.

Diskriminationstraining. Zu Beginn der Expositionsphase kann das Diskriminationstraining eingesetzt werden. Hierbei werden die Unterschiede zwischen aktuellen Auslösern, die die Patientin an das Trauma erinnern, und der damaligen Situation erarbeitet. In Situationen, in denen Reize die Patientin an traumatische Erfahrungen erinnern, soll sie sich besonders auf die Unterschiede zwischen damals und heute konzentrieren. Diese Unterschiede können sich beispielsweise auf die anwesenden Personen, deren Aussehen, die Umgebung und die eigenen Handlungsmöglichkeiten beziehen.

Gestufte Exposition. Zentrales Element dieser Phase ist die skills-assistierte Exposition gegenüber der heute am stärksten belastenden Erinnerung, dem Indexereignis. Diese erfolgt in mehreren Stufen: Zunächst berichtet die Patientin im Gespräch in der Vergangenheitsform von dem Ereignis. Anschließend fertigt sie einen schriftlichen Traumabericht an, den sie in der Sitzung vorliest. Im nächsten Schritt, der imaginativen Aktivierung, stellt die Patientin sich das Ereignis möglichst lebhaft vor und berichtet Schritt für Schritt von dem Geschehen.

\section{Die Exposition im Rahmen der DBT-PTSD folgt einem gestuften Vorgehen.}

Trauma-Netzwerk aktivieren. Um ein Kontextlernen zu ermöglichen, muss während der formalen Exposition das Trauma-Netzwerk aktiviert werden und gleichzeitig der Kontakt zur Gegenwart bestehen (vgl. Abb. 1). Um dieses Ziel zu erreichen, wird die Exposition unter der Zuhilfenahme von Skills durchgeführt, mit denen Patientin und Therapeut die emotionale Erregung modulieren. Hierzu erfragt der Therapeut in regelmäßigen Abständen die Stärke der Belastung sowie die gerade vorliegenden Gefühle. Bei Patientinnen mit hoher Dissoziationsneigung kann darüber hinaus das Ausmaß der Dissoziation erfragt werden.

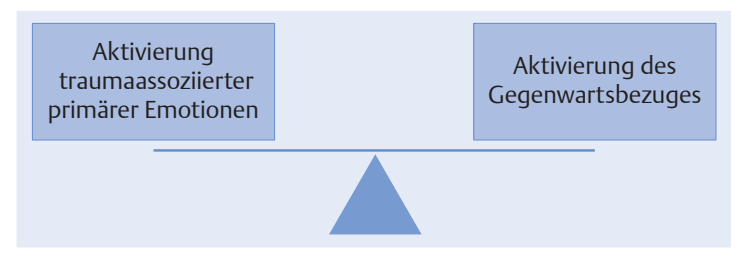

Abb. 1 Skills-assistierte Exposition.

Um das Trauma-Netzwerk stärker zu aktivieren, kann der Therapeut die Patientin bitten, die Augen zu schließen, das Geschehen in der Ich-Form und der Gegenwart zu beschreiben; er kann sich sensorische Details schildern lassen, sensorische Wahrnehmungen, z.B. bestimmte Geräusche und Gerüche bereitstellen, Gedanken und Gefühle verbalisieren sowie bestimmte Situationen in Zeitlupe durchgehen. Um den Gegenwartsbezug stärker zu aktivieren, kann der Therapeut im Gegensatz dazu sensorische Eindrücke aus der Gegenwart anbieten. Hierzu kann er z.B. die Patientin loben, sie bitten, die Augen zu öffnen, auf Geräusche hinweisen und ihr starke sensorische Reize anbieten,

\section{Tipp für die Praxis}

Während der Exposition muss der Realitätskontakt sichergestellt sein. Dazu wird während der Expositionsdurchführung das aktuelle Ausmaß der Belastung zwischen 0 und 100 eingeschätzt, der Therapeut wiederholt bei Bedarf das, was die Patientin sagt, und es werden Stressregulationsskills oder antidissoziative Skills eingesetzt. 
die für die gegenwärtige Situation stehen (z. B. Eisbeutel, Igelball).

Nachbereitung. An die Exposition schließt sich in der Regel eine kognitive Nachbereitung an, in der beispielsweise erneut aufgetretene Schuldgefühle besprochen werden.

Zwischen den Therapiestunden hören die Patientinnen zu Hause möglichst täglich eine Tonbandaufnahme der Sitzung an und führen mit deren Hilfe die Exposition selbstständig fort. Gegen Ende der Exposition werden Sätze zum Annehmen des Erlebten formuliert und in die Übungen integriert, wie z.B. „Ich wurde in der Kindheit missbraucht und das gehört zu meinem Leben, das werde ich nie vergessen können“.

Nachdem die Exposition gegenüber dem Indexereignis abgeschlossen ist, können bei Bedarf andere Ereignisse fokussiert werden. Gegebenenfalls kann das Imagery Rehearsal Training in die Behandlung integriert werden [35]. Dabei schreibt die Patientin einen belastenden Albtraum auf und verändert diesen zu einem guten Ende, welches sie dann eine Woche lang täglich imaginiert.

\section{Die Entfaltung des Lebens}

Neben der Förderung der Akzeptanz des Erlebten soll das therapeutische Team in dieser Behandlungsphase die Verbesserung der Lebensqualität im Fokus haben. Dabei geht es beispielsweise um eine Verbesserung des Selbstwerts sowie die Verhinderung einer erneuten
Viktimisierung. Je nach Lebenssituation der Patientin können Interventionen eingesetzt werden:

- zur Verbesserung des Körperbilds, z. B. durch Spiegeltraining

- zum Aufbau von Partnerschaften und Freundschaften

- zur Wiederaufnahme einer beruflichen Tätigkeit.

Hierzu können beispielsweise Skills zur Verbesserung der zwischenmenschlichen Fertigkeiten vermittelt werden, die die Patientin dabei unterstützen, in den Beziehungen zu anderen ihre eigenen Bedürfnisse zu äußern und sich von deren Forderungen abzugrenzen.

Behandlung im Team. Die DBT-PTSD wird begleitet von wöchentlicher Supervision und versteht sich auch ambulant - als Behandlung durch ein Team aus Therapeut, Supervisor und anderen Therapeuten. Die Patientin lernt den Supervisor und die anderen Behandler kennen. Der Behandlungsvertrag wird zu dritt mit dem Supervisor geschlossen, das erarbeitete Störungsmodell wird in der Supervisionsgruppe durch Patientin und Therapeut vorgestellt. Die Patientin weiß, dass die Supervisionsgruppe auch in den Therapieverlauf eingreifen kann. Dies geschieht z. B., wenn die Supervisionsgruppe den Eindruck gewinnt, dass Therapeut und Patientin gemeinsam vermeiden bzw. die formale Exposition hinauszögern.

Am Ende der Behandlung würdigt die Supervisionsgruppe bei einem letzten direkten Treffen die Arbeit der Patientin und des Therapeuten und überreicht der Patientin ein Zertifikat über den erfolgreichen Abschluss der Behandlung.

\section{Kasuistik}

\section{Fallvorstellung}

Spontan berichtete Symptomatik. Die

43-jährige Frau K. leidet aufgrund des sexuellen Missbrauchs durch ihren Vater und anderer physischer und sexueller Gewalterfahrungen, die sie während ihrer Ehe erleben musste, seit ca. 15 Jahren unter Intrusionen in Form von wiederkehrenden Bildern und Albträumen. Sie reagiert mit intensiver Angst und Belastung sowie körperlichen Symptomen (Zittern, Atemnot), wenn sie durch irgendetwas an den Missbrauch erinnert wird. Sie versucht, die Gedanken nicht zuzulassen und sich abzulenken. Aufgrund der starken Belastung kann sie vie- le Einzelheiten der traumatischen Übergriffe nicht erinnern. Sie fühlt sich wie „abgestorben“. Sie kann kaum Beziehungen eingehen, bleibt viel zu Hause. Bei starker Belastung fühlt sie sich z. T. wie „in Watte gepackt“. Außerdem leidet die Patientin unter Schlafstörungen und kann sich „nichts merken“. Bezüglich der Erlebnisse empfindet sie häufig Schuld sowie Scham, Ekel und Selbsthass. Frau K. fühlt sich außerdem nicht in der Lage, ihren Beruf auszuüben. Sie ist als Hauswirtschafterin in einer Klinik tätig und hat häufig mit sehr kranken Menschen zu tun. In diesen Situationen nimmt die traumaspezifische Sympto- matik und Belastung zu. Daher ist sie seit diesem Jahr arbeitsunfähig.

Ferner berichtet die Patientin von rasch einschießenden, wenig kontrollierbar erscheinenden Gefühlen. Häufig habe sie Druck, sich selbst durch Schneiden zu verletzen, sie führe es ca. 4- bis 5-mal jährlich oberflächlich aus, zuletzt vor 3 Monaten. Auch sei sie gereizt, sei gegenüber ihrem Partner und Arbeitskollegen verbal und physisch aggressiv (Gegenstände werfen). Bei innerer Anspannung hat Frau K. Essattacken und gibt viel Geld aus. In der Vorgeschichte ist es auch zu schädlichem Alkoholkonsum gekommen. Sie hat große Angst vor 
dem Alleinsein und berichtet von mehreren instabilen Beziehungen. Darüber hinaus ist sie seit ca. 4 Wochen sehr niedergeschlagen und traurig. Seit ihrer Kindheit erlebt sie immer wieder depressive Phasen.

Vorgeschichte. Die Patientin wuchs mit ihrer Schwester (+ 3 Jahre) im gemeinsamen Elternhaus auf. Der Vater wurde als sadistisch beschrieben, das frühere Verhältnis zu ihm sei gewaltvoll gewesen.

Die Mutter sei früher wie heute liebevoll gewesen, habe sich dem Vater jedoch stets unterworfen. Frau K. sei besonders von ihrem Vater bis zum 15. Lebensjahr emotional, physisch und sexuell misshandelt worden. Der Missbrauch habe nach einem Suizidversuch der Patientin geendet. Frau K. berichtet, dass sie als Kind unvorhersehbar und ohne für sie damals ersichtliche Gründe sehr hart bestraft worden sei. So sei sie als Kind sehr verschüchtert gewesen, habe sich nichts getraut. Sie sei darüber hinaus als Kind durch Fortlaufen, Stehlen und Lügen auffällig gewesen, sie habe „nur schlechte Erlebnisse“ gehabt und wenige Freunde. Frau K. berichtet von einer zurückliegenden Ehe, der Ehemann habe Alkohol- und Drogenprobleme gehabt, er habe versucht, sie zu erwürgen, und habe sie auch sexuell viktimisiert. In dieser Zeit habe Frau K. in einer Kneipe gearbeitet und viel Alkohol getrunken (ca. 8 Gläser Bier, ca. $16 \mathrm{cl}$ Schnaps täglich). Sie habe den Konsum ohne therapeutische Unterstützung eingestellt. Aus der Ehe entstand eine Tochter (heute 23 Jahre). Bei Behandlungsbeginn lebt Frau K. gemeinsam mit ihrem Partner in einer Mietwohnung. Zu ihrer Tochter hat sie regelmäßigen Kontakt, diese hat einen 1-jährigen Sohn. Zu ihren Eltern hat sie regelmäßigen Kontakt.

Diagnosen nach ICD-10. F43.1. Posttraumatische Belastungsstörung (PTBS), F33.1: Rezidivierende depressive Störung, derzeit mittelgradige depressive Episode, F60.31: Emotional-instabile Persönlichkeitsstörung, Borderline-Typus.

\section{Behandlungsverlauf}

Phase 1 (Commitment). Zu Beginn wurde mit Frau K. die aktuelle Symptomatik und der lebensgeschichtliche Hintergrund anhand der Lebenslinie erarbeitet sowie aktuelles Problemverhalten erfasst. Sie zeigte zu Beginn der Behandlung Dissoziation, selbstverletzendes Verhalten, Essanfälle, aggressives Verhalten sowie Alkoholabusus (in der Vorgeschichte). Gemäß der DBT-PTSD-Behandlungshierarchie formulierten wir die folgenden Behandlungsschwerpunkte:

- Standard-DBT:

- Stufe 1: Suizidalität

- DBT-PTSD:

- Stufe 1: dissoziative Symptomatik

- Stufe 3: Selbstverletzung, Alkoholabusus, Umgang mit Emotionen (v.a. Schuld, Scham, Ekel, Wut), Vermeidungsverhalten

- Stufe 4: Wiederherstellung der Arbeitsfähigkeit

Frau K. wurde über den Ablauf der DBTPTSD (Therapiephasen) aufgeklärt und in das computerbasierte Selbsthilfeprogramm zum Skills-Erwerb (Skills-Training) eingeführt. Therapeutin und Patientin schlossen einen speziellen Therapievertrag, in welchem Frau K. sich zu einem Non-Suizid-Versprechen verpflichtete und sich bereit erklärte, an der Aufgabe von Dissoziation, Selbstverletzungen, aggressivem Verhalten und Essanfällen zu arbeiten. Da Frau K. in der Vorgeschichte ein problematisches Trinkverhalten gezeigt hatte, einigten sich Therapeutin und Patientin ebenfalls auf ein Abstinenzversprechen, da sie davon ausgingen, dass die Vulnerabilität für erneutes problematisches Trinkverhalten durch die bevorstehende Behandlung steigen würde.

Phase 2 (Planung und Motivation). Zur Ableitung des Therapierationals erarbeiteten Therapeutin und Patientin ein individuelles Störungsmodell. Frau K. erhielt Psychoedukation zur Aufrechterhaltung der PTBS durch Vermeidungs- und Escape-Strategien und konnte das konfrontative Behandlungsrational ableiten. Mit der „Entscheidung für den neuen Weg“ erarbeitete Frau K. die kurz- und langfristigen Konsequenzen der Aufgabe von Vermeidung und stärkte somit ihre Entscheidung für die aktive Auseinanderset- zung mit den traumatischen Erlebnissen. Hierzu äußerte Frau K. allerdings zahlreiche Bedenken und Befürchtungen (z. B. "Ich werde mich wieder selbst verletzen, ich werde trinken, ich werde durchdrehen.“). Diesen Bedenken begegnete die Therapeutin, indem sie mit Frau K. im weiteren Teil der Behandlung Strategien zum Umgang mit Drang nach Problemverhalten besprach.

Phase 3 (Bearbeitung von traumabezogenen Escape-Strategien). Zunächst führte die Therapeutin die Tagebuchkarte ein, in der Frau K. den Drang nach Problemverhalten und andere Aspekte der Symptomatik täglich protokollieren sollte. Hierdurch konnte Frau K. schnell lernen, unterschiedliche Grade von Anspannung und Problemverhaltensdrang zu differenzieren. Zum besseren Verständnis des Problemverhaltens fertigte die Patientin Verhaltensanalysen zum letzten Suizidversuch und zur letzten Selbstverletzung an. Die Analyse des letzten Suizidversuchs ergab, dass die Patientin diesen als 15-jähriges Mädchen aus massiver Angst, Verzweiflung und Hoffnungslosigkeit heraus unternommen hatte. Die Konsequenzen waren, dass die Übergriffe durch den Vater aufhörten, jedoch auch, dass sie von der Familie ausgegrenzt wurde. Mit der Patientin wurden auch die kurz- und langfristigen Konsequenzen suizidaler Gedanken besprochen. Die Patientin konnte erkennen, dass es sich hierbei um einen Escape-Mechanismus handelte, um belastende emotionale Zustände zu beenden. Die Entscheidung zur Aufgabe dieser Strategie konnte gestärkt werden.

Um Alternativstrategien bei Anspannung zu erarbeiten, wurde die Patientin in die Grundlagen des Skills-Trainings eingeführt; verschiedene Skills-Module bei unterschiedlichen Anspannungsausprägungen wurden besprochen. Als Grundlage aller Fertigkeiten wurde Achtsamkeit benannt und erläutert. Sowohl während der Therapiestunde als auch zwischen den Stunden führte die Patientin regelmäßig Achtsamkeitsübungen durch. Um den Umgang mit Hochspannung zu verbessern, wurden Frühwarnzeichen (z. B. Kognition „Ich halte das 
nicht mehr aus“, Kribbeln im Körper) und Stresstoleranz-Skills erarbeitet. Besonders wichtig war es hier, auf das Phänomen von Dissoziation gesondert einzugehen. Die Patientin führte separate Dissoziationsprotokolle, um Dissoziation früher zu erkennen und zu unterbinden. Die hilfreichsten Stresstoleranz-Skills (z. B. Riechöl) sammelte Frau K. in einem Notfallkoffer.

Gleichzeitig zeigte sich auch schon in dieser Phase, dass die Patientin unter massiven Schuldgefühlen in Bezug auf den Missbrauch durch den Vater litt. Die Schuldgefühle und die Verleugnung des Geschehenen („Vielleicht bilde ich mir das alles nur ein.") konnten als EscapeStrategien aus dem starken Gefühl von Hilflosigkeit identifiziert werden. Hiernach wurden die Schuldkognitionen mithilfe kognitiver Techniken hinterfragt. Es wurde zunehmend deutlich, dass Frau K. ihre damaligen Handlungsmöglichkeiten überschätzte („Ich hätte mich nur mehr wehren müssen. “) und bei der Beurteilung ihres Verhaltens die Situation nur einseitig betrachtete. Beispielsweise ignorierte Frau K., dass es für sie als Kind keinerlei Möglichkeit gab, Aufmerksamkeit und Zuwendung durch die Eltern zu erfahren.

In diesem Rahmen begann die Patientin, ihre aktuell positive Beziehung zum Vater infrage zu stellen, sie könne „nur hassen oder nur lieben“ (Schwarz-Weiß-Denken). Im Rahmen eines Familienfestes mit den Eltern kam es dann zu massivem Alkoholkonsum. Diesen bearbeitete die Patientin mithilfe einer Verhaltensanalyse. Phase 4 (Exposition). Als erste Intervention sollte die Patientin das heute belastendste Ereignis (Indextrauma) als Hausaufgabe so ausführlich wie möglich auf- schreiben und in der folgenden Sitzung vorlesen. Anschließend wurden in den Sitzungen imaginatives Nacherleben und kognitive Nachbearbeitung eingesetzt. Frau K. war während der Expositionen hochbelastet; es mussten mehrfach antidissoziative Skills eingesetzt werden (z. B. Exposition während die Patientin sich auf einem Stepper bewegt), wodurch es der Patientin gelang, nicht zu dissoziieren.

In der kognitiven Nachbearbeitung wurden v. a. die Themen Schuld und Scham besprochen und mittels kognitiver Umstrukturierung bearbeitet. Eine weitere maladaptive Kognition bestand in der Annahme, dass angenehme Gefühle (in Form von sexueller Erregung) den Vater zu weiteren Übergriffen aufgefordert hätten. In diesem Zusammenhang erhielt die Patientin Psychoedukation zur sexuellen Erregung. Mithilfe kognitiver Techniken wurde an einer Neubewertung der angenehmen Gefühle gearbeitet. Die Patientin exponierte sich auch zwischen den Therapiesitzungen mit ihren Erinnerungen, indem sie die Audioaufnahmen der Sitzungen immer wieder anhörte. Die Belastung der Patientin nahm sehr langsam ab, die Dissoziationsneigung sank jedoch, sodass die Anwendung von Skills während der Expositionsübungen schrittweise ausgeschlichen werden konnte. Während der letzten Expositionssitzungen lernte die Patientin zunehmend auszuhalten, dass der Missbrauch für sie sowohl mit angenehmen als auch mit unangenehmen Gefühlen verknüpft war. Mithilfe von Strategien zur Emotionsregulation (besonders Emotionssurfen) und Achtsamkeit gelang es der Patientin, diese Spannung zu tolerieren und zunehmend Akzeptanz zu entwi- ckeln und Schuld- und Schamgefühle sowie Ekel, Ohnmachtserleben und Angst in Bezug auf die Erlebnisse abzubauen. Phase $\mathbf{5}$ (Entfaltung des Lebens). Im letzten Teil der Behandlung lag ein Schwerpunkt auf der Akzeptanzstärkung in Bezug auf das Vergangene. Die Patientin formulierte Sätze, die sie akzeptieren lernen wollte (z. B. „Ich wurde missbraucht, ja, das ist so.") und wiederholte diese im Rahmen einer Akzeptanzübung. Darüber hinaus äußerte die Patientin den Wunsch, ihre Arbeitstätigkeit wieder aufzunehmen. Frau K. wandte sich an den Arbeitgeber und besprach ihre Rückkehr. Frau K. wurde klar, dass sie sich in der Vorgeschichte bezüglich ihrer Leistungsfähigkeit häufig überfordert hatte und stellte somit einen Antrag auf Arbeitszeitverkürzung $(30 \mathrm{~h})$ und vereinbarte mit ihrem Vorgesetzten, Tätigkeiten mit weniger Belastung zu übernehmen.

\section{Behandlungsergebnis}

Am Ende der Behandlung berichtete die Patientin, dass sie sich mittlerweile deutlich „freier“ fühle. Sie traue sich mehr zu und könne mehr und mehr ein Leben nach „eigenen Vorstellungen führen“. Obwohl sie noch vereinzelt von Erinnerungen an die Gewalterfahrungen eingeholt werde, könne sie diese mit Distanz betrachten und habe nicht mehr den Drang, die Erinnerungen zu vermeiden. Problemverhalten in Form von Suizidgedanken, Selbstverletzungen, Dissoziation, Essattacken, Alkoholkonsum oder Kaufen treten überhaupt nicht mehr auf, es bestehe auch kein Drang mehr. Sie habe das Gefühl, „ganz gut alleine klar zu kommen“, ihr mache die Zukunft keine Angst mehr (s.a. Tab. 4 und Abb. 2).

\section{Empirische Ergebnisse zur Wirksamkeit der DBT-PTBS}

Pilotstudie. Steil und Kollegen untersuchten im Rahmen einer Prä-Post-Studie die Wirksamkeit einer 12wöchigen DBT-PTSD an 29 Patientinnen mit PTBS nach sexuellem Missbrauch in der Kindheit und zumindest einer anderen komorbiden Störung nach DSM-IV (z.B. einer komorbiden schweren Depression, Anorexia oder Bulimia nervosa, Substanzmissbrauch oder BorderlinePersönlichkeitsstörung) [8]. Die Zahl der Ausschlusskriterien wurde dabei auf ein Minimum begrenzt: Schizophrenie, mentale Retardation oder eine schwere Psychopathologie, die eine Behandlung in einem anderen Setting indizierte (wie z. B. ein Body-Mass-Index unter 16 oder akute Suizidalität) wurden ausgeschlos- 


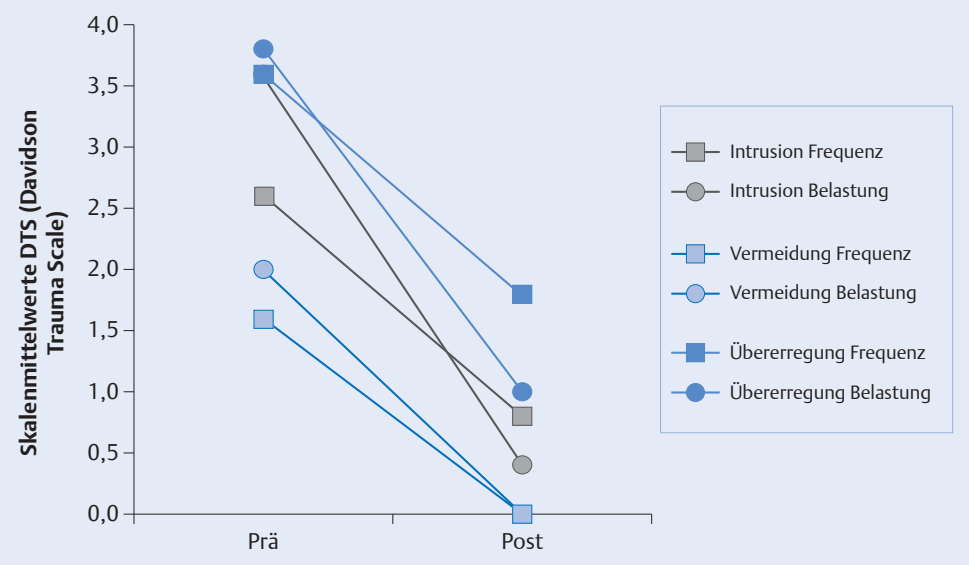

Abb. 2 Behandlungsergebnis der traumaspezifischen Symptomatik bei Frau K.

\section{Tabelle 4}

Behandlungsergebnisse für die DBT-PTSD bei Frau K.

\begin{tabular}{|c|c|c|}
\hline & $\begin{array}{l}\text { Behandlungs- } \\
\text { beginn }\end{array}$ & $\begin{array}{l}\text { Behandlungs- } \\
\text { ende }\end{array}$ \\
\hline CAPS & & \\
\hline $\begin{array}{l}\text { alle traumatischen } \\
\text { Ereignisse }\end{array}$ & 96 & 22 \\
\hline Index-Trauma & 97 & 22 \\
\hline BDI & 52 & 2 \\
\hline SCL-90 (T-Wert) & 80 & 56 \\
\hline $\begin{array}{l}\text { Borderline-Symptom- } \\
\text { liste }\end{array}$ & $\begin{array}{l}\mathrm{PR}=90 \text { Befind- } \\
\text { lichkeit }=10 \%\end{array}$ & $\begin{array}{l}\text { PR }=46 \text { Befind- } \\
\text { lichkeit }=70 \%\end{array}$ \\
\hline
\end{tabular}

sen. Die Wirksamkeit wurde mithilfe verschiedener Selbstbeurteilungsinstrumente vor der Behandlung sowie 6 und 12 Wochen danach erfasst:

- Posttraumatische Diagnoseskala (PDS)

- Symptom-Checklist-90-Revised (SCL-90-R)

- Beck-Depressionsinventar (BDI)

- State-Trait-Anxiety Inventory (STAI).

Die Patientinnen waren im Schnitt 35,4 Jahre alt. Die Zeit zwischen dem Missbrauch und dem Behandlungsbeginn betrug im Schnitt 27,4 Jahre. Die Patientinnen hatten im Schnitt 3,8 Diagnosen der Achsen I oder II des DSM-IV (90\% eine schwere Depression, $45 \%$ eine Persönlichkeitsstörung, 38\% eine Essstörung und 14\% eine substanzassoziierte Störung). Die Effektstärken für den Vergleich zwischen Prä- und Follow-up-Messung

\section{Tabelle 5}

Effektstärken DBT-PTSD im Vergleich zu einer

Wartelistenkontrollgruppe.

\begin{tabular}{|ll|}
\hline Verwendetes Instrument/Symptomatik & Hedge's g \\
\hline $\begin{array}{l}\text { Clinician Administered PTSD Scale } \\
\text { (PTBS im Fremdurteil) }\end{array}$ & 1,60 \\
\hline $\begin{array}{l}\text { Posttraumatic Diagnostic Scale } \\
\text { (PTBS im Selbsturteil) }\end{array}$ & 0,98 \\
\hline $\begin{array}{l}\text { Borderline Symptom List } \\
\text { (Borderline-Symptome im Selbsturteil) }\end{array}$ & 0,52 \\
\hline $\begin{array}{l}\text { Fragebogen zu dissoziativen Symptomen } \\
\text { (Dissoziation im Selbsturteil) }\end{array}$ & 0,50 \\
\hline $\begin{array}{l}\text { Beck-Depressionsinventar II } \\
\text { (Depression im Selbsturteil) }\end{array}$ & 0,70 \\
\hline
\end{tabular}

waren groß mit Cohen's d=1,22 (PDS), 1,04 (BDI), 0,52 (SCL-90-R) und 0,71 (STAI-Trait).

Randomisierte kontrollierte Studie. Bohus und Kollegen untersuchten die Wirksamkeit einer 12-wöchigen stationären DBT-PTSD im Vergleich mit einer Wartelistenbedingung an 74 Patientinnen mit PTBS mit schwerer Störung der Emotionsregulation nach sexuellem Missbrauch in der Kindheit und vergleichbarer Symptomatik [7]. Bei 74\% der Patientinnen beinhaltete der sexuelle Missbrauch auch Penetration. Die Patientinnen waren im Schnitt 35 Jahre alt, hatten im Schnitt 3 Diagnosen der Achse I des DSM-IV. 44,6\% der Patientinnen hatten eine Borderline-Persönlichkeitsstörung, über 79\% eine schwere depressive Episode. Es handelte sich also um eine schwer kranke Patientengruppe. Tab. 5 zeigt die Effektstärken für den Vergleich zwischen Behandlungs- und Wartelistengruppe zum letzten Messzeitpunkt 6 Monate nach Therapieende. In den verwendeten Selbst- und Fremdbeurteilungsmaßen zur PTBS-Symptomatik zeigten sich große bis sehr große Effektstärken zugunsten der DBT-PTSD.

Zwölf Wochen nach Therapieende zeigten deutlich mehr Patientinnen der Behandlungsgruppe eine Remission der PTBS als in der Wartelistengruppe (38,9\% vs. 10,5\%). Weder die Diagnose einer Borderline-Persönlichkeitsstörung noch die Anzahl der entsprechenden Symptome war bedeutsam assoziiert mit dem Therapieerfolg. 


\section{Fazit zur Wirksamkeit der DBT-PTSD}

Mit der DBT-PTSD gibt es für die stationäre Behandlung von schwer kranken PTBS-Patientinnen nach Missbrauchserfahrungen ein strukturiertes, die besonderen Probleme gestörter Emotionsregulation berücksichtigendes Behandlungsprogramm, das auch Patientinnen mit wiederkehrender Suizidalität oder Selbstverletzung, komorbider Borderline-Persönlichkeitsstörung bzw. weiterer komorbider Symptomatik sicher und effektiv behandeln kann. Der Vergleich mit anderen schon länger eingeführten Interventionen steht bislang noch aus.

Derzeit wird in einer großen multizentrischen Studie (Release-Projekt) in den Zentren Berlin, Frankfurt und Mannheim eine ambulante Form der DBT-PTSD verglichen mit einer der meist beforschten Psychotherapien der PTBS - der Cognitive Processing Therapy [36].

\section{Kernaussagen}

Die Hauptsymptome der PTBS sind Intrusionen, Vermeidungsverhalten, traumaspezifische dysfunktionale Kognitionen und Übererregung.

Nach multipler Traumatisierung in der Kindheit und Jugend entwickeln Patientinnen häufig zusätzlich zu der PTBS-Kernsymptomatik Störungen der Emotionsregulation. In der Vergangenheit fehlten auf die Bedürfnisse und Besonderheiten dieser Patientengruppe angepasste evidenzbasierte Behandlungsprogramme.

Die in den letzten 10 Jahren entwickelte DBT-PTSD ist bislang die einzige Intervention, die eine gezielte traumafokussierte und formale Exposition beinhaltende Struktur für Patientinnen mit PTBS und Störung der Emotionsregulation aufweist.

Der Behandlung der DBT-PTSD liegt eine für DBT-Interventionen typische dynamische Behandlungshierarchie zugrunde, bei der die Behandlung einem klaren Algorithmus folgt, der dem Thera- peuten bzw. dem behandelnden Team hilft zu entscheiden, in welcher Reihenfolge problematische Verhaltensweisen oder Störungsbereiche fokussiert und behandelt werden sollen. Die Hierarchisierung der Behandlung hat das Ziel, auch bei schwer kranken Patientinnen möglichst bald mit der formalen Exposition beginnen zu können, die das Kernelement der Behandlung darstellt. Die DBT-PTSD besteht aus 5 Behandlungsphasen: Commitment, Planung und Motivation, Bearbeitung von traumabezogenen Escape-Strategien, Exposition: Reduktion des Wiedererlebens traumabezogener Gefühle und Entfaltung des Lebens. Die DBT-PTSD wird begleitet von wöchentlicher Supervision und versteht sich als Behandlung durch ein Team aus Therapeut, Supervisor und anderen Therapeuten.

Die Wirksamkeit der DBT-PTSD wurde in bislang 2 Studien belegt. 


\section{Über die Autorinnen}

\section{Regina Steil}

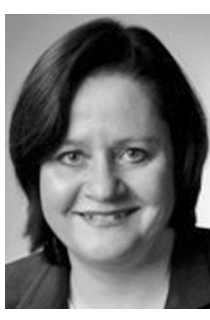

Dr. rer. nat. Dipl.-Psych. Jahrgang 1965. Psychologische Psychotherapeutin und Supervisorin. Wissenschaftliche Geschäftsführerin der Verhaltenstherapie-Ambulanz an der Abteilung Klinische Psychologie und Psychotherapie der Goethe-Universität Frankfurt, Mitherausgeberin der Zeitschrift Trauma und Gewalt, Past President der Deutschsprachigen Gesellschaft für Psychotraumatologie.

\section{Clara Dittmann}

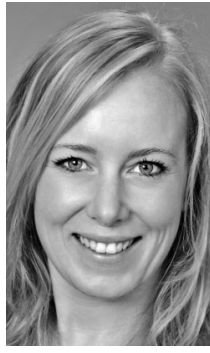

Dipl.-Psych. Jahrgang 1985. Bis 2011 Studium der Psychologie in Mainz und Valencia. Seit 2012 Weiterbildung zur Psychologischen Psychotherapeutin (Schwerpunkt Verhaltenstherapie). Seit 2014 klinische und wissenschaftliche Mitarbeiterin am Institut für Psychologie der Goethe-Universität Frankfurt im Bereich Traumatherapie.

\section{Simone Matulis}

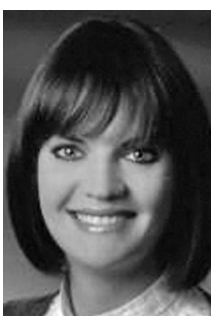

Dipl.-Psych. Jahrgang 1983. Seit 2012 Psychologische Psychotherapeutin (Schwerpunkt Verhaltenstherapie) und Fachkundenachweis zur Behandlung von Kindern und Jugendlichen. Zusatzqualifikation „Spezielle Psychotraumatherapie (DeGPT)“. Bis 2014 wissenschaftliche Mitarbeiterin in der Abteilung Klinische Psychologie und Psychotherapie und administrative Leitung der Trauma-Ambulanz der Goethe-Universität Frankfurt. Tätigkeit in der Weiterbildung von ärztlichen und psychologischen Psychotherapeuten und Kinder- und Jugendlichenpsychotherapeuten.

\section{Meike Müller-Engelmann}

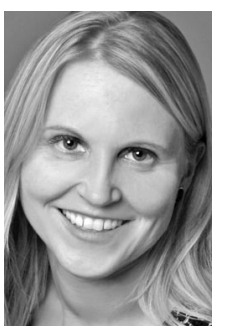

Dr. rer. nat. Dipl.-Psych. Jahrgang 1978. Seit 2010 Psychologische Psychotherapeutin (Schwerpunkt Verhaltenstherapie). Zusatzqualifikation in Psychotraumatologie (DeGPT) und DialektischBehavioraler Therapie. Seit 2013 wissenschaftliche Mitarbeiterin am Institut für Psychologie der Goethe-Universität Frankfurt. Supervisoren- und Dozententätigkeit im Bereich Traumatherapie.

\section{Kathlen Priebe}

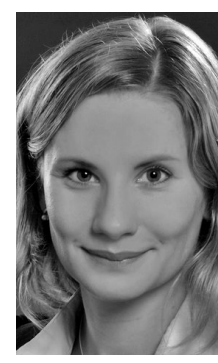

Dipl.-Psych. Jahrgang 1977. Psychologische Psychotherapeutin. Seit 2005 Klinische und wissenschaftliche Mitarbeiterin der Klinik für Psychosomatik und Psychotherapeutische Medizin am Zentralinstitut für seelische Gesundheit in Mannheim. Seit 2014 Wissenschaftliche Mitarbeiterin am Institut für Psychologie der Humboldt-Universität zu Berlin. Supervisoren- und Dozententätigkeit im Bereich Traumatherapie.

Interessenkonflikt: Regina Steil gibt Supervision, Schulungen und Vorträge gegen Honorar zur DBT-PTSD. Meike Müller-Engelmann gibt Supervision und Schulungen gegen Honorar zur DBT-PTSD. Kathlen Priebe gibt Supervision, Schulungen und Vorträge gegen Honorar zur DBT-PTSD.

Clara Dittmann und Simone Matulis geben an, dass kein Interessenkonflikt besteht.

\section{Korrespondenzadresse}

Dr. Regina Steil

Abteilung Klinische Psychologie und Psychotherapie

Goethe-Universität Frankfurt

Varrentrappstraße 40-42

60486 Frankfurt

E-Mail: steil@psych.uni-frankfurt.de 


\section{Literatur}

1 Harned MS, Rizvi SL, Linehan MM. Impact of co-occurring posttraumatic stress disorder on suicidal women with borderline personality disorder. Am J Psych 2010; 167: 1210-1217

2 Pagura J, Stein MB, Bolton JM et al. Comorbidity of borderline personality disorder and posttraumatic stress disorder in the U.S. population. J Psychiatr Res 2010; 44: 1190-1198

3 Ztlotnick C, Johnson DM, Yen S et al. Clinical features and impairment in women with borderline personality disorder (BPD) with posttraumatic stress disorder (PTSD), BPD without PTSD, and other personality disorders with PTSD. J Nerv Ment Dis 2003; 191: 706-713

4 Priebe K, Steil R, Kleindienst $\mathrm{N}$ et al. Psycholoische Behandlung der Posttraumatischen Belastungsstörung nach sexuellem Missbrauch: Eine Übersicht. Psychother Psychosom Med Psychol 2012; 62: 5-17

5 Neuner F. Stabilisierung vor Konfrontation in der Traumatherapie - Grundregel oder Mythos? Verhaltenstherapie 2008; 18: $108-118$

6 Rosner R, Henkel C, Ginkel K et al. Was passiert nach der stationären Stabilisierung mit komplex traumatisierten PTBPatientinnen? Die Bedeutung von Stabilisierung und Konfrontation für die Behandlung traumatisierter. Frauen Z Psychiatr Psych Ps 2010; 58: $127-135$

7 Bohus $M$ et al. DBT for PTSD after Childhood Sexual Abuse in Patients with and without Borderline Personality Disorder: A Randomised Controlled Trial. Psychother Psychosom 2013; 82: $221-233$

8 Steil R, Dyer A, Priebe K et al. Dialectical behavior therapy for posttraumatic stress disorder related to childhood sexual abuse: a pilot study of an intensive residential treatment program. J Trauma Stress 2011; 24: $102-106$

9 Diagnostic and Statistical Manual of Mental disorders. 5th: ed. Washington, DC: American Psychiatric Association; 2013

10 Herman JL. Complex PTSD: a syndrome in survivors of prolonged and repeated trauma. J Trauma Stress 1992; 5: 377 391

11 Maercker A, Brewin CR, Bryant RA et al. Diagnosis and classification of disorders specifically associated with stress: proposals for ICD-11. World Psychiatry 2013; 12: 198-206

12 Bradley R, Greene J, Russ E et al. A multidimensional metaanalysis of psychotherapy for PTSD. Am J Psych 2005; 162 : 214- 227

13 Bisson J, Andrew M. Psychological treatment of post-traumatic stress disorder (PTSD) (Review). Cochrane Database Syst Rev 2007; 3: CD003388

14 Bisson JI, Ehlers A, Matthews R et al. Psychological treatments for chronic post-traumatic stress disorder. Systematic review and meta-analysis. Br J Psychiatry 2007; 190: 97 - 104

15 Clarke SB, Rizvi SL, Resick PA. Borderline personality characteristics and treatment outcome in cognitive-behavioral treatments for PTSD in female rape victims. Behav Ther 2008; 39: $72-78$

16 Feeny NC, Zoellner LA, Foa EB. Treatment outcome for chronic PTSD among female assault victims with borderline personality characteristics: A preliminary examination. J Pers Disord 2002; 16: $30-40$

17 Hembree EA, Cahill SP, Foa EB. Impact of personality disorders on treatment outcome for female assault survivors with chronic posttraumatic stress disorder. J Pers Disor 2004; 18 : $117-127$

18 McDonagh A, Friedman M, McHugo G et al. Randomized trial of cognitive-behavioral therapy for chronic posttraumatic stress disorder in adult female survivors of childhood sexual abuse. J Consult Clin Psychol 2005; 73: 515
19 Foa EB, Keane TM, Friedman MJ, Cohen JA, Hrsg. Effective treatments for PTSD: practice guidelines from the International Society for Traumatic Stress Studies. New York: Guilford Press; 2008

20 Benedek DM, Friedman MJ, Zatzick D et al. Guideline Watch: Practice guideline for the treatment of patients with acute stress disorder and posttraumatic stress disorder. Psychiatry Online 2009: Doi: 10.1176/appi.books.9780890423479. 156498

21 Ursano RJ, Bell C, Spencer E et al. Practice guideline for the treatment of patients with acute stress disorder and posttraumatic stress disorder. Psychiatry Online 2004: Doi: 10.1176/appi.books.9780890423363.52257

22 National Collaborating Centre for Mental Health. Post-traumatic stress disorder: The management of PTSD in adults and children in primary and secondary care. London: Gaskell and the British Psychological Society; 2005

23 Forbes D, Creamer M, Phelps A et al. Australian guidelines for the treatment of adults with acute stress disorder and posttraumatic stress disorder. Aust NZ J Psychiatry 2007; 41: 637 648

24 Flatten G, Gast U, Hofmann A et al. S3-Leitlinie Posttraumatische Belastungsstörung. Trauma \& Gewalt 2011; 3: $202-210$

25 Cloitre M, Courtois CA, Charuvastra A et al. Treatment of complex PTSD: Results of the ISTSS expert clinician survey on best practices. J Trauma Stress 2011; 24: 615-627

26 Cloitre M, Koenen KC, Cohen LR et al. Skills training in affective and interpersonal regulation followed by exposure: a phase-based treatment for PTSD related to childhood abuse. J Consult Clin Psychol 2002; 70: 1067 - 1074

27 Cloitre M, Stovall-McClough KC, Nooner K et al. Treatment for PTSD related to childhood abuse: A randomized controlled trial. Am J Psych 2010; 167: 915-924

28 Stoffers JM, Voll BA, Rucker G et al. Psychological therapies for people with borderline personality disorder. Cochrane Database Syst Rev 2012; 18: CD005652; Doi: 10.1002/14651858. CD005652.pub2

29 Harned MS, Linehan MM. Integrating dialectical behavior therapy and prolonged exposure to treat co-occurring borderline personality disorder and PTSD: Two case studies. Cogn Behav Pract 2008; 15: 263-276

30 Linehan MM. Dialektisch-Behaviorale Therapie der BorderlinePersönlichkeitsstörung. München: CIP; 1996

31 Harned MS, Korslund KE, Foa EB et al. Treating PTSD in suicidal and self-injuring women with borderline personality disorder: Development and preliminary evaluation of a dialectical behavior therapy prolonged exposure protocol. Behav Res Ther 2012; 50: $381-386$

32 Harned MS, Korslund KE, Linehan MM. A pilot randomized controlled trial of Dialectical Behavior Therapy with and without the Dialectical Behavior Therapy Prolonged Exposure protocol for suicidal and self-injuring women with borderline personality disorder and PTSD. Behav Res Ther 2014; 55: 7 17

33 Bohus M, Wolf M. Interaktives Skillstraining für BorderlinePatienten. Manual zur CD-ROM für die therapeutische Arbeit. Stuttgart: Schattauer Verlag; 2009

34 Priebe K, Dyer A. Metaphern, Geschichten und Symbole in der Traumatherapie. Göttingen: Hogrefe; 2014

35 Krakow B, Zadra A. Clinical management of chronic nightmares: imagery rehearsal therapy. Behav Sleep Med 2006; 4: $45-70$

36 Resick PA, Monson CM, Chard KM. Cognitive processing therapy: Veteran/military version. Washington, DC: Department of Veterans Affairs; 2007 


\section{CME.thieme.de}

\section{CME-Fragen}

CME-Teilnahme

- Viel Erfolg bei Ihrer CME-Teilnahme unter http://cme.thieme.de

- Diese Fortbildungseinheit ist 12 Monate online für eine CME-Teilnahme verfügbar.

- Sollten Sie Fragen zur Online-Teilnahme haben, unter http://cme.thieme.de/hilfe finden Sie eine ausführliche Anleitung.

1

Welche Aussage ist richtig? Die Hauptsymptome einer PTBS nach DSM-5 sind:
A Intrusionen, Übererregung, vermindertes Selbstwertgefühl und Albträume

B Intrusionen, Vermeidungsverhalten, Übererregung sowie negative Veränderungen in Kognitionen und Stimmung

C Albträume, Vermeidungsverhalten, Übererregung, negative Veränderung in Kognitionen und Stimmung, Dissoziation

D Störung der Emotionsregulation, Vermeidungsverhalten, Übererregung, negative Veränderung in Kognitionen und Stimmung

E Vermeidungsverhalten, Übererregung, Retraumatisierung, vermindertes Selbstwertgefühl

2

Welche Aussage zu bisherigen Therapieansätzen ist falsch?
A Traumafokussierende kognitiv-behaviorale Behandlungsverfahren und Eye Movement Desensitization and Reprocessing (EMDR) zeigen große Effekte auf die posttraumatische Symptomatik.

B In den Leitlinien der International Society for Traumatic Stress Studies wird eine traumafokussierende kognitiv-behaviorale Behandlung empfohlen.

C Es existiert eine Vielzahl gut etablierter und bezüglich ihrer Wirksamkeit überprüften Verfahren zur Behandlung der PTBS bei Patienten mit schwerer Störung der Emotionsregulation.

D Die vorhandenen Daten sprechen dafür, dass die Elemente der Standard-DBT bei der Mehrheit der BPS-Patientinnen mit komorbider PTBS nicht ausreichen.

E In der DBT+PE erhalten Patientinnen während einer einjährigen Standard-DBT zusätzlich traumafokussierende Expositionssitzungen.

3

Welche Aussage zur Behandlungshierarchie ist richtig?

\section{4}

Welche Aussage zur Emotionsregulation in der DBT-PTSD ist richtig?

\section{5}
A Sobald die Exposition begonnen wurde, spielt die Hierarchie keine Rolle mehr.
B Selbstverletzungen sind immer vorrangig zu behandeln.
C Die Patientinnen entscheiden, welche Probleme wann im Verlauf der Therapie zu besprechen sind.
D Die Hierarchie bleibt die ganze Behandlung hindurch bestehen.
E Dissoziative Symptome werden bereits in den Stufen der Standard-DBT abschließend behandelt.

Was ist Diskriminationstraining?
A Die Skills zur Stresstoleranz sind der einzige Bestandteil der Emotionsregulation.
B Die Emotionsregulation spielt vor der Expositionsbehandlung keine Rolle.
C Die Interventionen zur Emotionsregulation beinhalten Skills zum Umgang mit Dissoziation, Skills zur Stresstoleranz und Maßnahmen zur Reduktion der emotionalen Verwundbarkeit.
D Die Patientin muss vor Beginn der formalen Exposition Belastungen optimal reduzieren können.
E Die Emotionsregulation beinhaltet ausschließlich die Behandlung dissoziativer Symptome.

A Das Diskriminationstraining dient dazu, zwischen den einzelnen Stufen der emotionalen Anspannung zu unterscheiden.

B Das Diskriminationstraining steht am Ende der Behandlung.

C Das Diskriminationstraining dient der Unterscheidung verschiedener Traumata.

D Beim Diskriminationstraining werden die Unterschiede zwischen aktuellen Auslösern, die die Patientin an das Trauma erinnern, und der damaligen Situation erarbeitet.

E Das Diskriminationstraining ist Bestandteil der Albtraumbehandlung. 


\section{CME-Fragen}

Dialektisch-behaviorale Therapie der PTBS bei Patientinnen mit schwerer Störung der Emotionsregulation

\section{6}

Welche Aussage zum Beginn der formalen Exposition ist richtig?
A Vor Beginn der Exposition muss eine umfassende Stabilisierung erfolgen und die Patientin darf keine Selbstverletzungen mehr zeigen.

B Auch wenn eine Patientin noch überzeugt davon ist, schuld an dem traumatischen Ereignis zu sein, kann mit der Exposition begonnen werden.

C Die Patientin sollte auf die Zunahme der Belastung während der Expositionsbehandlung vorbereitet werden.

D Die Patientin darf in den letzten Monaten nicht akut suizidal gewesen sein.

E Vor Beginn der Exposition dürfen keine dissoziativen Symptome mehr vorkommen.

\section{7}

Was wird in der formalen Exposition in der DBT-PTSD fokussiert?
A Es ist nicht vorgeschrieben, mit welchem Trauma begonnen wird.

B Traumata aus der Kindheit werden in jedem Fall vorrangig behandelt.

C Es müssen insgesamt mindestens 3 traumatische Ereignisse bearbeitet werden.

D Die Exposition beginnt mit dem Indexereignis.

E Wenn neben sexueller Gewalt andere traumatische Ereignisse vorliegen, dann wird mit diesen begonnen, da die sexuelle Gewalt mit zu starken Belastungen verbunden ist.

8

Welche Aussagen stimmen bezüglich der formalen Exposition in der DBT-PTSD?
A Die formale Exposition erfolgt skillsbasiert, um eine gute Balance zwischen der Aktivierung des Traumanetzwerks und der Aktivierung des Gegenwartsbezugs herstellen zu können.

B Es wird kein Traumabericht geschrieben.

C Die Exposition wird unterbrochen, sobald Selbstverletzungen vorkommen.

D Die Behandlungshierarchie ist aufgehoben, sobald mit der Exposition begonnen wurde.

E Die formale Exposition wird aufgrund der damit verbundenen hohen Belastung ausschließlich in den Sitzungen durchgeführt.

\section{9}

Welche Aussage bezüglich der letzten Behandlungsphase in der DBT-PTSD ist richtig?
A Die Interventionen der letzten Behandlungsphase sind genau festgelegt.

B Auch in der letzten Behandlungsphase sollte die Exposition fortgesetzt werden.

C Im Fokus dieser Behandlungsphase steht die Verbesserung der Lebensqualität.

D Das Spiegeltraining ist obligatorisch.

E Dissoziative Symptome werden erst in der letzten Behandlungsphase bearbeitet.

10

Welche Aussage zur Studienlage ist richtig?
A Zur Untersuchung der Wirksamkeit der DBT-PTSD liegen eine Pilotstudie und eine randomisierte kontrollierte Studie vor. Die Effektstärken sind groß bis sehr groß.

B Zur Untersuchung der Wirksamkeit der DBT-PTSD liegen keine empirischen Studien vor.

C Die DBT-PTSD ist weniger wirksam als bisherige Interventionen.

D Zur Untersuchung der Wirksamkeit der DBT-PTSD liegen 4 Studien vor.

E Die Effektstärken sind klein bis mittelgroß. 